La revue La revue pour l'histoire du CNRS

POUR L'HISTOIRE DU CNRS $\quad 15 \mid 2006$

CNRS et Université

\title{
Le financement de l'enseignement supérieur et de la recherche en Allemagne
}

Manfred Heinemann

\section{OpenEdition}

Journals

Édition électronique

URL : https://journals.openedition.org/histoire-cnrs/513

DOI : 10.4000/histoire-cnrs.513

ISSN : 1955-2408

Éditeur

CNRS Éditions

Édition imprimée

Date de publication : 3 novembre 2006

ISBN : 978-2-271-06452-3

ISSN : 1298-9800

\section{Référence électronique}

Manfred Heinemann, « Le financement de l'enseignement supérieur et de la recherche en Allemagne », La revue pour l'histoire du CNRS [En ligne], 15 | 2006, mis en ligne le 23 novembre 2008, consulté le 20 mai 2021. URL : http://journals.openedition.org/histoire-cnrs/513 ; DOI : https://doi.org/10.4000/ histoire-cnrs. 513

Ce document a été généré automatiquement le 20 mai 2021.

Comité pour l'histoire du CNRS 


\title{
Le financement de l'enseignement supérieur et de la recherche en Allemagne
}

\author{
Manfred Heinemann
}

1 La situation actuelle est la suivante. Le Bund souhaite se retirer du financement des établissements universitaires en Allemagne et se limiter à l'avenir, dans le contexte européen et international en mutation, à subventionner la recherche. C'est tout le système de financement public conjoint par le Bund et les Länder - caractéristique du système d'enseignement supérieur et de recherche en Allemagne - qui est ainsi remis en cause.

2 Cela va bien au-delà de la rupture avec la tradition du financement conjoint, établie depuis l'immédiat après-guerre, et marque le début d'un nouveau système. Cela pourrait entraîner l'accroissement des disparités régionales déjà existantes entre le Sud et le Nord dans les capacités éducatives et scientifiques des Länder. Les disparités salariales au sein du personnel enseignant et des chercheurs devraient également augmenter suite au transfert aux Länder des compétences relatives aux fonctionnaires et à leurs traitements.

3 Si l'on ajoute aux conséquences du «braindrain» du nord-est vers le sud-ouest, le déplacement du potentiel scientifique que cela induit pour l'économie, on entrevoit de nouvelles et profondes transformations des structures au sein du système d'enseignement supérieur et de recherche dans les années à venir. Le cas de Berlin, comme pôle universitaire et scientifique, illustre remarquablement ces évolutions. À Berlin, science et technologie sont présentées, en terme de marketing, comme les principaux atouts pour l'avenir. ${ }^{1}$ Le pôle scientifique d'Adlershof (dans l'Est de Berlin) est désormais le plus important d'Allemagne. Cependant, l'étude économique Deutschland 2010 classe Berlin au $266^{\mathrm{e}}$ rang, comme un pôle de taille moyenne. ${ }^{2}$ Il faut dire que cette étude mesure ici non pas le nombre des «cerveaux» dans les établissements d'enseignement supérieur, mais le rapport des coûts salariaux à la productivité. Cela montre à quel point les investissements en matière d'enseignement 
supérieur et de recherche ne produisent guère d'effets sur le plan économique, si l'environnement industriel ne croît pas de manière corrélative. Les effets que la régénération du système scientifique des pays de l'Europe centrale et orientale - en cours grâce aux moyens mis à disposition par l'Union européenne - produiront dans quelques années en Allemagne ne sont guère débattus. Car c'est en recrutant une part croissante d'étudiants venus de ces pays et d'autres pays non-européens que les établissements universitaires allemands parviennent à combler les manques, par rapport à leurs capacités d'accueil, alors que le nombre des étudiants allemands chute. ${ }^{3}$

On en connaît les causes: la structure démographique d'une société allemande vieillissante, malgré l'augmentation du pourcentage d'étudiants par génération, ${ }^{4}$ conduira à une réduction des besoins en matière d'éducation et d'enseignement supérieur. En raison de l'endettement croissant du Bund et des Länder, les pôles universitaires et scientifiques sont mis en concurrence avec d'autres secteurs, nécessitant eux aussi des investissements financiers, et se retrouvent ainsi en voie de privatisation ou de déperdition qualitative. Le transfert à des fondations de droit public de l'université de Göttingen - ainsi que d'autres établissements universitaires en BasseSaxe - illustre cette évolution de façon exemplaire. ${ }^{5}$ La délocalisation de l'industrie vers l'Europe centre-orientale, la Chine ou d'autres pays émergents explique aussi ce processus. Le développement des capacités de recherche dans ces pays a lui aussi des conséquences. Dans certains domaines de recherche, les besoins sont chancelants, si bien qu'il n'est pas facile de dégager de nouveaux créneaux et l'établissement de «spécialités» en Allemagne ou en Europe fait l'objet d'une grande concurrence. Identifier ces nouveaux créneaux entraîne des dépenses considérables, ce que montrent les rapports et le nombre incalculable d'instances, essentiellement financées par l'argent public, qui cherchent à les définir. La part de ces stratégies d'identification et des frais administratifs augmente dans les dépenses globales. Les programmes européens d'aide à la recherche sont toujours moins performants au vu du rapport des frais engagés et de l'utilité.

5 Le tableau 1 donne un aperçu des dépenses quasi stables des Länder et des communes en Allemagne entre 1995 et 1999. Les dépenses de recherche et de développement (R\&D) restent elles aussi à peu près constantes. 
Tableau 1

DÉPENSES SCIENTIFIQUES DE LA RFA

en millions d'euros

\begin{tabular}{|c|c|c|c|c|c|c|}
\hline Sources de financement & 1991 & 1993 & 1995 & 1997 & 1999 & 2001 \\
\hline \multicolumn{7}{|l|}{ 1. Budgets publics } \\
\hline Bund & 10273 & 10491 & 10374 & 10122 & 10229 & 11141 \\
\hline Länder & 13569 & 15518 & 16673 & 17055 & 17360 & 18423 \\
\hline Communes & 165 & 157 & 159 & 162 & 149 & 192 \\
\hline Total 1 & 24007 & 26166 & 27206 & 27339 & 27738 & 29756 \\
\hline 2. Organismes scientifiques* & 859 & 840 & 1007 & 1229 & 1396 & 1600 \\
\hline Total $1+2$ & 24866 & 27006 & 28213 & 28568 & 29134 & 31356 \\
\hline \multicolumn{7}{|l|}{ 3. Milieu économique } \\
\hline $\begin{array}{l}\text { Activité industrielle } \\
\text { et commerciale }\end{array}$ & 23935 & 23973 & 24733 & 27036 & 32411 & 35095 \\
\hline Fondations et dons & 317 & 317 & 317 & 307 & 330 & 330 \\
\hline Total 3 & 24252 & 24290 & 25050 & 27343 & 32741 & 35425 \\
\hline Total $1+2+3$ & 49118 & 51296 & 53263 & 55911 & 61875 & 66781 \\
\hline
\end{tabular}

Tableau 1 : Dépenses scientifiques de la RFA en millions d'euros.

7 Les dépenses similaires de 2002 s'élèvent à près de 19 milliards d'euros (pour 1999, voir le tableau 1). ${ }^{6}$ Ainsi, les Länder et les communes ont-ils réduit leurs dépenses pour l'enseignement supérieur et la recherche.

Publiquement, on ne parle guère des fermetures d'établissements universitaires et scientifiques. Les effets au niveau régional en seraient trop négatifs. Aussi, l'argumentation est-elle différente. Par le biais des initiatives d'excellence, on cherche, sur le plan financier, à mieux préparer à la concurrence internationale un certain nombre d'établissements choisis. Les établissements qui ne sont pas subventionnés dans ce cadre des initiatives d'excellence deviendront à plus ou moins long terme des institutions de second rang. Les flux d'étudiants étrangers pourront peut-être atténuer, mais non stopper, le processus en cours de réduction des capacités. Selon les disciplines, la situation du marché de l'emploi est plus ou moins favorable pour les jeunes diplômés. Si le besoin en ingénieurs augmente à un moment donné, un nombre important de médecins qualifiés quittent l'Allemagne dans le même temps. ${ }^{7}$ La référence au marché européen du travail ne sera guère un atout pour les établissements universitaires provincialisés. En règle générale, les effets régionaux positifs de l'enseignement supérieur et de la recherche ne se traduisent qu'à long terme par le développement de centres technologiques en relation avec l'industrie.

Il n'y a plus de développement des infrastructures d'enseignement et de recherche en Allemagne. Les besoins sont couverts. Désormais, au cours des vingt prochaines années, c'est sous l'effet de la concurrence que se transformera la carte de l'enseignement supérieur. Chaque Land cherche désespérément à percevoir les tendances par le biais de rapports d'expertises sur les évolutions démographiques et économiques régionales. Pour les Länder du Nord et de l'Est, la situation est plus difficile que dans ceux du Sud et de l'Ouest, plus prospères. Partout, la restructuration et la réorientation interne des 
cursus sont à l'ordre du jour. Partout, l'on procède à des évaluations et l'on réduit les dépenses.

La situation parlementaire actuelle s'est transformée: Bund et Länder ont d'autres priorités. En Allemagne, depuis 10 ans environ, on ne considère plus comme allant de soi le financement de l'enseignement supérieur et de la recherche à hauteur de plusieurs milliards d'euros. Désormais chaque poste compte.

11 Les positions différentes du Bund et des Länder seront amenées à se différencier encore davantage. D'un point de vue historique, le Bund, depuis la fondation de la RFA en 1949, a eu longtemps une influence croissante. En 1950, il récupéra la plupart des institutions de recherche de l'ancien Reich ; conformément aux accords de Königstein, ${ }^{8}$ il soutint le développement des institutions scientifiques dans les Länder, renouvela et refonda le système d'aide à l'enseignement supérieur et à la recherche (la DFG), le DAAD (office d'Échanges universitaires Allemands avec l'étranger) etc. et, par l'adjonction de l'article 91 b à la Loi Fondamentale en 1969 - du temps de la première Grande Coalition -, il s'assura une sphère d'influence prédominante dans la politique scientifique.

\section{Tableau 2}

DÉPENSES DES LANDËR ET DES COMMUNES POUR LES ÉTABLISSEMENTS D'ENSEIGNEMENT SUPÉRIEUR, LES CLINIQUES UNIVERSITAIRES

ET LA RECHERCHE EXTRA-UNIVERSITAIRE

(recherche et développement)

en millions d'euros

\begin{tabular}{lrrrrr}
\hline Land & $\mathbf{1 9 9 5}$ & $\mathbf{1 9 9 7}$ & 1999 & $\mathbf{2 0 0 1}$ & $\mathbf{2 0 0 2}$ \\
\hline Bade-Wurtemberg & 2225,5 & 2194,3 & 2392,8 & 2487,1 & 2538,1 \\
\hline Bavière & 2509,8 & 2632,7 & 2614,5 & 2759,3 & 2769,5 \\
\hline Berlin & 1598,1 & 1487,5 & 1398,7 & 1480,3 & 1525,2 \\
\hline Brandebourg & 329,0 & 364,8 & 359,7 & 318,6 & 327,8 \\
\hline Brême & 195,0 & 193,3 & 223,6 & 251,6 & 280,2 \\
\hline Hambourg & 543,9 & 558,6 & 596,4 & 559,6 & 598,8 \\
\hline Hesse & 1220,9 & 1203,4 & 1261,5 & 1344,0 & 1364,8 \\
\hline Mecklembourg & 333,4 & 386,5 & 402,0 & 378,9 & 382,9 \\
\hline Basse-Saxe & 1358,4 & 1355,5 & 1466,0 & 1886,3 & 1840,5 \\
\hline Rhénanie du Nord Westphalie & 3069,3 & 3291,7 & 3256,6 & 3485,2 & 3979,4 \\
\hline Rhénanie-Palatinat & 576,5 & 615,5 & 611,9 & 664,4 & 622,1 \\
\hline Sarre & 210,4 & 199,3 & 208,9 & 219,5 & 215,8 \\
\hline Saxe & 1026,8 & 1101,9 & 1069,7 & 1145,1 & 1083,2 \\
\hline Saxe-Anhalt & 549,9 & 576,0 & 570,2 & 576,1 & 589,4 \\
\hline Schleswig-Holstein & 524,7 & 495,3 & 473,8 & 484,6 & 484,1 \\
\hline Thuringe & 559,4 & 561,2 & 602,9 & 574,6 & 560,8 \\
\hline Total & 16831,0 & 17217,5 & 17509,2 & 18615,2 & 19162,6 \\
\hline Dont dépenses R\&O* & 15813,0 & 15682,0 & 15800,0 & 16744,0 & 16299,0 \\
\hline
\end{tabular}

- pardes Lander et des counumincs

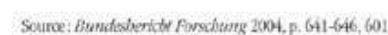

12 Tableau 2: Dépenses des Landër et des communes pour les établissements d'enseignement supérieur, les cliniques universitaires et la recherche extrauniversitaire (recherche et développement) en millions d'euros.

En 2002, la totalité des dépenses d'enseignement supérieur et de recherche en RFA se monta à 68,1 milliards d'euros, ce qui correspond à une augmentation de près de $10 \%$ par rapport à 1999 (cf. tableau 1). La part du financement par le milieu économique atteint désormais 36,2 milliards d'euros, représentant désormais $53 \%$ du total des dépenses. La part de l'État (Bund et Länder) s'élève à 31,9 milliards d'euros, soit seulement $47 \%{ }^{9}$. Les dépenses publiques s'accroissent en valeur absolue depuis le début du millénaire, mais, globalement, leur part recule. C'est ainsi que les politiques ont fini par atteindre l'un de leurs objectifs : l'accroissement de la prise en charge par 
les milieux économiques. Au cours des prochaines années, l'augmentation des recettes par financement privé (les droits d'inscription des étudiants etc.) devrait permettre de consolider les établissements universitaires, dans la mesure où le ministre des Finances $\mathrm{du}$ Land leur accordera de telles ressources.

Trois quarts de l'ensemble des dépenses universitaires et scientifiques en Allemagne ont été attribuées en 2002 aux établissements d'enseignement supérieur et à peine un quart aux établissements de recherche extra-universitaires. Cette répartition est constante depuis longtemps. Il se peut que désormais les ministres des Finances, qui consolident leurs budgets par des mesures de restrictions, soient contraints de toucher davantage encore aux établissements universitaires pour continuer à miser sur la recherche et le développement comme force motrice de la consolidation économique.

L'aide à la recherche : une tâche conjointe du Bund et des Länder

Plus d'un quart des aides de l'État dans le domaine de la recherche et du développement extra-universitaires (montant total de 31,6 milliards de DM en 1999) reviennent à des institutions scientifiques d'importance supra-régionale sur la base de l'article 91 b de la Loi Fondamentale. En voici la répartition :

\section{Tableau 3}

\section{AIDE CONJOINTE Ȧ LA RECHERCHE PAR LE BUND ET LES LÄNDER (RECHERCHE ET DÉVELOPPEMENT) en millions d'euros} 1999

\begin{tabular}{lrrr}
\hline Établissements & Bund & Länder & Total \\
\hline Max-Planck-Gesellschaft & 391,2 & 403,0 & 794,2 \\
\hline Deutsche Forschungsgemeinschaft & 601,7 & 425,0 & 1026,8 \\
\hline Fraunhofer-Gesellschaft & 209,6 & 45,5 & 255,1 \\
\hline Programme des Académies ${ }^{\star}$ & 18,4 & 18,4 & 36,8 \\
\hline Centres Helmholtz $^{\star \star}$ & 1409,8 & 175,9 & 1585,7 \\
\hline Liste Bleue $^{*}$ & 321,5 & 312,5 & 634,0 \\
\hline Académie allemande Leopoldina, Halle a.d.S. & 2,0 & 0,5 & 2,5 \\
\hline Total & $\mathbf{2 9 5 4 , 3}$ & $\mathbf{1 3 8 0 , 7}$ & $\mathbf{4 3 3 5 , 0}$
\end{tabular}

- Soutien à des projets

- * Voir http://www.helmholtz.de/Downloads/1_Wir_uber_uns/Forschungszentren.html

Tableau 3: Aide conjointe à la recherche par le Bund et les Länder (recherche et développement) en millions d'euros.

La réforme du fédéralisme

Dans les négociations en vue de la coalition, la SPD et la CDU se sont entendues sur la réforme du fédéralisme. ${ }^{10}$ La tâche conjointe " construction d'institutions d'éducation supérieure » doit être supprimée, mais l'aide pour les équipements lourds et les projets d'excellence au niveau national est maintenue et transférée dans le cadre de la tâche conjointe " aide à la recherche ». La tâche conjointe " programmation éducative » doit elle aussi être supprimée. Une fois perçus les aspects négatifs d'un tel démantèlement, une partie du Parlement s'est préparée à une certaine confrontation avec le gouvernement de coalition. ${ }^{11}$ Il y aura un débat parlementaire, mais il est peu vraisemblable que cela change le cours des choses.

L'aide à l'enseignement supérieur et à la recherche comme tâche conjointe 

système universitaire et scientifique est-allemand, l'article $91 \mathrm{~b}$ de la Loi Fondamentale ( « coopération en matière de programmation de l'enseignement et de la recherche ») avait permis de lier étroitement le Bund et les Länder pour le financement de l'enseignement supérieur et de la recherche. Cet article stipule : « sur le fondement de conventions, la Fédération et les Länder peuvent coopérer pour la planification de l'enseignement et pour la promotion de centres et de projets de recherche scientifique d'intérêt supra-régional. La répartition des coûts est réglée dans la convention ». Aujourd'hui, la réforme prévue le remet en cause. Il ne restera à l'avenir que la «promotion de projets de recherche d'intérêt supra-régional ». D'aucuns craignent déjà qu'il n'en résulte la paupérisation accrue du paysage universitaire et scientifique au Nord et à l'Est de l'Allemagne. Le résultat de la première sélection (un $2^{\mathrm{e}}$ " tour » est prévu) dans le concours «initiative d'excellence - universités d'élite pour l'Allemagne » est éloquent : sur 319 candidatures, 36 établissements universitaires ont été retenus et parmi eux, aucun ne se trouve dans la partie orientale de l'Allemagne. ${ }^{12}$ Comme la compétence cadre du Bund dans le domaine universitaire, prévue par l'article 751 a de la Loi Fondamentale est supprimée et que, de surcroît, une législation dérogatoire a été décidée par la Grande Coalition dans le domaine universitaire (art. 72, $\S 3, n^{\circ} 6$ ), il pourrait en résulter des restrictions financières toujours plus drastiques pour l'enseignement supérieur et la recherche, combinées à la poursuite de la réduction des capacités universitaires et scientifiques.

«L'égalité des conditions de vie » : un modèle dépassé pour le financement de l'enseignement supérieur et de la science?

Ce n'est sans doute qu'après de laborieux débats constitutionnels que l'on saura si l'article 72 conduira un jour à une nouvelle législation fédérale en matière universitaire et scientifique afin d'établir "l'égalité des conditions de vie", inscrite dans la Loi Fondamentale. Aujourd'hui, on reconnaît plutôt comme fait établi l'inégalité des conditions de vie entre les Länder de la République Fédérale. ${ }^{13}$ C'est ainsi que le président de la RFA, Horst Köhler, a déclaré dans une interview au magazine FOCUS, le 13 septembre 2005 : « si un salarié ne trouve pas dans sa région d'emploi correspondant à ses capacités, il doit se décider : ou bien il va là où il voit des chances d'atteindre ses objectifs professionnels ; ou bien il accorde sciemment priorité à vivre dans sa région ". Un discours de 2000 du ministre-président de l'époque de Rhénanie du NordWestphalie, devenu par la suite ministre fédéral de l'Économie, Wolfgang Clement, reflète à quel point la reconnaissance des inégalités régionales est aussi ancrée dans la pensée de la SPD. ${ }^{14}$

Autrement dit, la question est de savoir dans quelle mesure l'ouverture du marché européen de l'enseignement et de la science conditionne l'accroissement des inégalités. Les diplômés de l'enseignement supérieur sont-ils des précurseurs d'une redistribution de la carte universitaire et scientifique européenne dans le mouvement de migration en Europe? Les pérégrinations intra-européennes des détenteurs du capital intellectuel n'ont-elles pas déjà leurs racines dans les transformations en cours du financement du système universitaire et scientifique ? Ou bien, pour reprendre les propos de Köhler : «Il nous faut rétablir au sein de la société l'acceptation d'une reconnaissance particulière des performances, pour donner à la solidarité sociale une base économique solide ». Concrètement: l'élite de l'élite doit-elle continuer d'aller s'implanter en Bavière ou en Bade-Wurtemberg?

La revue pour l'histoire du CNRS, 15 | 2006 
Renouvellement du concept d'aide ou de soutien

Une présentation des établissements publics d'enseignement supérieur et de recherche sous le seul angle de leur financement ne permet pas d'appréhender correctement la situation du système allemand où l'État, jusque-là dominant, est en train de se retirer. Aujourd'hui, il faut prendre en compte les investissements dans l'enseignement et la recherche, émanant des familles, à partir de leur capital privé, et ceux résultant d'un capital corporatif, des fondations par exemple. S'y ajoute la privatisation, en cours depuis plusieurs années, de tâches éducatives incombant naguère à l'État ou aux communes.

Les voies vers un « espace économique fondé sur la science »

\section{Tableau 4}

\begin{tabular}{|c|c|c|c|c|}
\hline Secteurs tinanç̧ant & 1991 & 1995 & 1997 & 1999 \\
\hline Bund & 5625,23 & 8460,35 & 8212,37 & 8574,36 \\
\hline Indice & 100 & 97 & 94 & 99 \\
\hline en \% R\&D & 22,2 & 20,5 & 19,1 & 18,1 \\
\hline Länder & 6232,65 & 7352,89 & 7474,06 & 7597,8 \\
\hline Indice & 100 & 118 & 120 & 122 \\
\hline en \% R\&D & 15,9 & 17,8 & 17,4 & 16,0 \\
\hline État (Bund+Länder) & 11857,88 & 15813,24 & 15686,43 & 16172,16 \\
\hline Indice & 100 & 106 & 105 & 108 \\
\hline en \% R\&D & 38,1 & 38,3 & 36,5 & 34,1 \\
\hline Milieux économiques & 24004,64 & 25330,42 & 27153,69 & 31038,48 \\
\hline Indice & 100 & 106 & 113 & 129 \\
\hline en $\%$ R\&D & 61,4 & 61,4 & 63,2 & 65,5 \\
\hline Institutions privées* & 195,31 & 103,75 & 141,12 & 152,36 \\
\hline Indice & 100 & 53 & 72 & 78 \\
\hline en \% R\&D & 0,5 & 0,3 & 0,3 & 0,3 \\
\hline \multirow[t]{2}{*}{ Total Dépenses R\&D } & 36057,83 & 41247,41 & 42981,24 & 47363 \\
\hline & 100 & 105 & 110 & 121 \\
\hline en \% du Revenu national brut & 2,59 & 2,31 & 2,30 & 2,42 \\
\hline
\end{tabular}

Tableau 4 : Dépenses de recherche et développement (R\&D) de la RFA par instances de financement en millions d'euros.

En 1999, le total des dépenses pour la recherche et le développement incombant au Bund et aux Länder se montait à 31,6 milliards de DM (16,15 milliards d'euros). ${ }^{15}$ Observons maintenant la situation dans un Land. Le plan de financement à moyen terme (2005-2009) du Land de Thuringe (qui gère à peine $2 \%$ des dépenses de recherche et de développement de la RFA) laisse clairement apparaître quel est, pour cette région, l'objectif à atteindre conformément aux accords européens : Selon la décision du Conseil européen de Barcelone (2002), dans le cadre de l'application de la stratégie de Lisbonne, les dépenses de recherche et de développement doivent être portées « de 2 à $3 \%$ du PIB » pour créer l'espace économique fondé le plus fortement et le plus globalement possible sur la science. « Pour cela, la part du secteur économique privé dans les dépenses de recherche et de développement, au niveau européen, doit être porté de $56 \%$ à deux tiers. Un tiers des dépenses doit provenir du secteur public. Les dépenses de recherche et développement se montent actuellement à 2,5\% du PIB en Allemagne et à $2,1 \%$ en Thuringe ${ }^{16}{ }^{16}$ 
24 Selon ce plan, sans la croissance du secteur économique privé, l'objectif ne peut être atteint. Pour la Thuringe, il y aurait un fort besoin de rattrapage des dépenses de recherche et de développement par l'économie régionale, car la part de cette dernière se monte à peine à $55 \%$, contre $70 \%$ en moyenne pour l'ensemble de l'Allemagne. « La capacité d'autofinancement des petites et très petites entreprises de Thuringe ne suffit souvent pas à mener les activités de recherche et de développement de manière suffisante ». Il n'y a pas de " grosses entreprises », moteurs de la recherche appliquée et du développement. «Il faudrait renforcer les activités de recherche appliquée dans les établissements scientifiques et universitaires de Thuringe ainsi que les interconnexions entre l'économie et la science d'une part, et les entrepreneurs entre eux de l'autre (création de réseaux et de groupes)».

On ne voit pas pourquoi de tels objectifs ne pourraient être atteints par l'application de ce schéma à un niveau supra régional. Fondamentalement, on comprend bien que ce modèle « d'espace économique fondé sur la science » est un défi valable pour toutes les disciplines. Cela se reflète depuis des années dans la dépendance croissante de la recherche universitaire à l'égard des "Drittmittel» (subventions allouées par des organismes tiers) qui, selon le modèle envisagé devraient venir - idéalement- du secteur privé, ce que ce dernier, déjà mis à contribution d'une manière importante pour le financement des dépenses de recherche et de développement, ne fait pas. Ceux qui ont développé ce modèle attendent que des milliards d'euros viennent du secteur économique privé. Aussi est-il logique de diminuer la charge fiscale des entreprises pour stimuler dans le sens voulu la restructuration des fonds de recherche et développement. La logique de ce modèle conduit aussi à réduire proportionnellement l'aide à la recherche et au développement à partir de financements publics, si les valeurs moyennes pour l'Allemagne sont atteintes en Thuringe. Jusqu'à présent on disait plutôt «en augmentant les dépenses publiques de recherche et de développement, on espère aussi induire une hausse des dépenses privées dans ce secteur, car les deux sont dans un rapport complémentaire ».

La subsidiarité comme principe directif européen

Dans ce contexte s'affirme l'idée de subsidiarité, qui a marqué la pensée allemande depuis l'émergence de l'État moderne vers 1800 . Elle renvoie aussi à la tradition prussienne de retrait de l'État face à l'activité socio-économique. La formulation classique du principe de subsidiarité se retrouve à l'alinéa 79 de l'encyclique Quadragesimo anno promulguée par le pape Pie XI en 1931 dans le contexte de l'expansion des totalitarismes communiste, fasciste et national-socialiste. ${ }^{17}$ En ce sens, les subventions dans le domaine de la recherche et du développement ne sont justifiées qu'aussi longtemps que les acteurs économiques ne s'en chargent pas eux-mêmes.

Après plusieurs sessions, la convention européenne a intégré le principe de subsidiarité dans l'Union..$^{18}$ Aussi faut-il en conclure que l'aide au secteur de recherche et de développement est déjà soumise à un contrôle particulier, afin d'assurer la dégressivité des aides accordées. Étant donné qu'en 2000, l'économie allemande, avec presque deux tiers des dépenses totales à hauteur de 60,7 milliards de DM (31,04 milliards d'euros), était le plus grand pourvoyeur de fonds de la recherche allemande, ${ }^{19}$ elle va poursuivre la coopération en matière de recherche avec des partenaires scientifiques et économiques. L'accroissement du volume des dépenses de l'économie pour la recherche a conduit corrélativement à la prise en compte fiscale de la recherche universitaire en 
Allemagne, exempte jusqu'alors de TVA. Ainsi l'État, avec ces revenus fiscaux, peut-il à son tour financer sa politique structurelle.

Les objectifs de la politique scientifique et le primat de la promotion de l'économie

Günther Gloser, alors député SPD au Bundestag et secrétaire d'État au ministère fédéral des Affaires étrangères depuis 2005, avait établi le lien entre aide à l'économie et recherche : «La TVA doit être portée de 16 à $19 \%$ au $1^{\text {er }}$ janvier 2007. Nous avons au moins obtenu le report d'un an. C'est plus qu'une mesure cosmétique : à partir de 2006 nous investirons dans la dynamisation de l'économie. Pour renforcer l'innovation, l'investissement, la croissance et l'emploi, nous dépenserons 25 milliards d'euros jusqu'en 2009; dans le domaine de l'innovation 6 milliards d'euros : À ce titre, il faut compter le relèvement de la part de la recherche et du développement à $3 \%$ du PIB d'ici 2010 (''État pour un tiers et l'économie pour les deux tiers), l'initiative d'excellence pour le renforcement de la recherche universitaire ainsi que pour le pacte recherche et innovation. $»^{20}$

En 2000, le montant des financements Recherche et Développement versés par l'Union européenne à l'Allemagne dans le cadre des programmes de recherches atteignait environ $4 \%$ de l'aide publique à la recherche en Allemagne. Dans certains domaines, comme les biotechnologies ou les techniques de l'information, la part a pu s'élever à 10 ou $20 \%$. Cette année, de nouvelles aides ont été proposées dans le cadre des programmes COST (coopération européenne dans le domaine de la recherche scientifique et technique) et EUREKA (initiative pour une coopération technologique renforcée en Europe). Au titre des programmes européens de formation SOCRATES et LEONARDO, l'Allemagne a reçu 3 milliards d'euros pour la période 2000-2005.

Les dépenses de recherche et de développement reflètent la baisse constante de la part de l'État, une part minime et constante venues des institutions privées à but non lucratif et des fondations reconnues d'utilité publique et l'apport nettement croissant du milieu économique. Aussi, toutes les mesures qui renforcent cette tendance sontelles prioritaires. Le temps où l'État, et avant tout le Bund, finançait l'innovation en Allemagne est révolu depuis longtemps déjà. Toutes les exceptions qui perdurent encore par suite de l'intégration de l'Allemagne de l'Est à ces modèles seront financées de manière séparée par des primes de solidarité. Mais les dépenses d'enseignement et de recherche des Länder de l'Est restent proportionnellement inférieure à leur population. S'ils se dépeuplent encore, comme on le pronostique, il faudra reconsidérer la situation.

\section{NOTES}

1. Cf. http://www.berlinews.de/archiv-2004/3623.shtml

2. Cf. http://www.managermagazin.de/unternehmen/standortstudie/

0,2828,365956-7,00.html

3. Cf. http://www.wissenschaftweltoffen.de/2002/1/1/8/5 
4. Le rapport fédéral sur la recherche de 2004 exige dans le paragraphe 2.2 : «Favoriser les ressources humaines ", de : commencer en particulier dans les écoles; augmenter le pourcentage des étudiants ; grâce aux universités d'élite, attirer les meilleurs cerveaux vers l'Allemagne; renforcer le soutien aux jeunes chercheurs ; brain gain au lieu brain drain ; encourager la mobilité du personnel. http://www.bmbf.de/pub/bufo2004.pdf 5. Christoph Conrads, „Entbürokratisierung und Entstaatlichung - Die Georgia Augusta in Stiftungsträgerschaft", In Bildung und Erziehung 57 (2004) 3, p. 327-343.

6. Bundesbericht Forschung, 2004, S. 174.

7. Brain Drain - Brain Gain. Eine Untersuchung über internationale Berufskarrieren von Beate Backhaus, Lars Ninke, Albert Over 2002. http://www.dfg.de/ wissenschaftliche_karriere/focus/2003/doppelkarriere_paare/download/ braindrain_studie.pdf

8. La « clé de Königstein » règle la répartition entre les Länder dans les financements communs, notamment ceux de

la DFG, de la MPG et des établissements de la Société Leibniz :

http://www.blkbonn.de/koenigsteiner_schluessel.htm

9. Rapport fédéral sur la recherche, 2004, p. 174.

10. Contrat de coalition entre la CDU, la CSU et la SPD du 11 novembre 2005. http:// www.spd.de/servlet/PB/show/1589444/111105_Koalitionsvertrag.pdf

11. http://dip.bundestag.de/btd/16/002/1600223.pdf

12. Voir : http://www.dfg.de/aktuelles_presse/reden_stellungnahmen/2006/ download/exin_gemeinsame_pm.pdf

13. Voir le rapport du congrès (21/22 octobre 1999) : 10 Jahre nach dem Fall der Mauer : Gleichwertige Lebensverhältnisse " oder bleibende Ungleichheit", http:// www.gesis.org/dauerbeobachtung/sozialindikatoren/Veranstaltungen/Berichte/ Lebensverh.htm

14. Voir : http://www.wsws.org/de/2000/mai2000/clem-m06.shtml Voir aussi :

Christopher Jencks, Chancengleichheit, Reinbek bei Hamburg, 1973.

15. Bundesbericht Forschung, 2000, S. 53.

16. Voir :http://www.thueringen.de/de/tfm/haushalt/plan/uc21/u_start.html

17. Handwörterbuch des politischen Systems der Bundesrepublik, 2000 [Manfred Spieker: Subsidiarität] Voir http://www.bpb.de/wissen/ 06701398267020876160471336933330,0,0,Subsidiarit\%E4t.html

18. Voir : http://register.consilium.eu.int/pdf/de/02/cv00/00286d2.pdf

19. Bundesbericht Forschung, 2000, p. 53.

20. http://www.campact.de/mwst/infos/antw_gloser

\section{RÉSUMÉS}

« Depuis des mois, les spécialistes mettent en garde contre le retrait de l'État fédéral (Bund) de la politique d'éducation. Enfin, ils sont entendus». Quel est le problème? Dans le cadre de la réforme du fédéralisme en Allemagne, décidée par le nouveau gouvernement de grande coalition CDU-SPD, la répartition des compétences en matière d'éducation et de recherche doit être 
révisée. Quelles sont les craintes ? Les présidents des établissements d'enseignement supérieur, la Deutsche Forschungsgemeinschaft (DFG) et même le Deutscher Wissenschaftsrat, tous voient se profiler à l'horizon une provincialisation susceptible d'affaiblir davantage encore le système allemand d'enseignement et de recherche.

"Since several months, specialists have warned about the pull out of the Federal state from Education politics. Finally, they've been heard ». What is the problem? In a context of the reform of federalism in Germany decided by the new great coalition CDU-SPD in power, the repartition of skills about Education and scientific research must be reviewed. What are the fears? The presidents of universities and superior schools (including the Deutsche Forschungsgemeinschaft (DFG) and the Deutscher Wissenschaftsrat) are afraid of an even less centralized system that could weaken the Education and research German systems.

\section{AUTEUR}

\section{MANFRED HEINEMANN}

Manfred Heinemann est historien, professeur à l'université de Hanovre, où il dirige le Zentrum für Zeitgeschichte von Bildung und Wissenschaft. Il a été membre et président du conseil d'administration de l'université technique de Chemnitz (1997-2005) et viceprésident (1991-1993) de la commission pour l'enseignement supérieur de Saxe, chargée de la rénovation universitaire après la Wende. 